

Watling, J.R. and Asenov, A. and Barker, J.R. (1998) Efficient hole transport model in warped bands for use in the simulation of $\mathrm{Si} / \mathrm{SiGe}$ MOSFETs. In, International Workshop on Computational Electronics, 19-21 October 1998, pages pp. 96-99, Osaka, Japan.

http://eprints.gla.ac.uk/3034/ 


\title{
Efficient Hole Transport Model in Warped Bands for Use in the Simulation of Si/SiGe MOSFETs
}

\author{
J.R.Watling*, A. Asenov and J.R. Barker \\ Device Modelling Group \\ Department of Electronics and Electrical Engineering \\ University of Glasgow, Glasgow G12 8LT, UK \\ Tel: +44 1413304792 , Fax: +441413304907 \\ *E-mail: J.Watling@elec.gla.ac.uk
}

An analytical geometric model for the valence band in strained and relaxed $\mathrm{Si}_{1-\mathrm{x}} \mathrm{Ge}_{\mathrm{x}}$ is presented, which shows good agreement with a 6-band $\mathbf{k} \cdot \mathbf{p}$ analysis of the valence band. The geometric model allows us to define an effective mass tensor for the warped valence band structure. The model also has applications in the study of III-V semiconductors, and could aid in the interpretation of cyclotron resonance experiments in these bands. A warped three-band Monte Carlo simulation has been developed based on this model making use of the efficient calculation of trajectory dynamics that is made possible through the use of such a model. The calculated transport characteristics show good agreement with the available experimental data.

\section{Introduction}

Transport of holes in strained $\mathrm{Si} / \mathrm{Si}_{\mathrm{l}-\mathrm{x}} \mathrm{Ge}_{\mathrm{x}}$ layers has gained great interest recently because of the proven benefits of such structures for use in high-speed p-MOSFETs. In such layers the hole mobility may become comparable to the electron mobility, thus strained $\mathrm{Si} / \mathrm{S}_{1-\mathrm{x}} \mathrm{Ge}_{\mathrm{x}}$ hole transport layers are extremely attractive for BiCMOS applications, with the possibility of velocity overshoot occurring in submicron devices.

The modelling of holes using accurate, analytical bands is not as advanced as that for electrons due to the complex quartic nature of their energy surfaces, even in the unstrained case. Many authors have either used highly simplified models [1], or used full band Monte Carlo [2-4] models. However, the use of either type of model is unattractive for device simulation since the former does not reproduce the correct symmetry of the bandstructure, while the latter significantly slows down practical device simulations, necessitating the use of large powerful computers. In order to develop an efficient Monte Carlo for the modelling of hole transport in modern semiconductor devices we have developed a valence band model based on algebraic geometric invariants of the constant energy surfaces. Section two of this paper describes the geometric valence band model we have developed, and the third section presents results on the transport properties.

\section{Geometric Valence Band Model}

There have been previous attempts to develop analytical models for the warped valence bands in silicon and germanium, notably the model presented by Dresselhaus, Kip and Kittel [5]. However, this model is only valid at low energies as it neglects the interaction between the spin-split off band $\mid 1 / 2,1 / 2>$ and the heavy $\mid 3 / 2,3 / 2>$ and light $\mid 3 / 2,1 / 2>$ hole bands. This interaction is important at room temperature in silicon due to the close proximity of the spin-split off band $(44 \mathrm{meV})$ to the heavy and light hole bands. Also the model does not reproduce the correct symmetry of the strained system.

We have calculated the bandstructure using an exact diagonalisation of the 6-band $\mathbf{k} \cdot \mathbf{p}$ Hamiltonian [6,7] based on the method of Kane. Strain is included using the perturbation Hamiltonian of Pikus and Bir [8]. The 6-band $\mathbf{k} \cdot \mathbf{p}$ model has been used since it has been shown to give a good approximation to the bandstructure calculated using the pseudopotential method [9]. Examples of the bandstructures calculated for $\mathrm{S}_{1-\mathrm{x}} \mathrm{Ge}_{\mathrm{x}}$ (both relaxed and strained) using $\mathbf{k} \cdot \mathbf{p}$ are shown in Fig.1 and Fig.2 for an energy of $40 \mathrm{meV}(\sim 3 / 2$ $\mathrm{k}_{\mathrm{B}} \mathrm{T}, \mathrm{T}=300 \mathrm{~K}$ ).

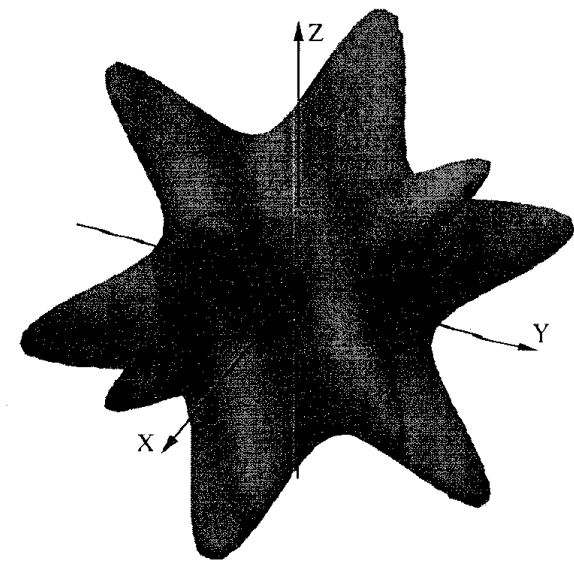

Fig.1: The heavy hole $\mid 3 / 2,3 / 2>$ band in relaxed $\mathrm{Si}_{0.7} \mathrm{Ge}_{0.3}$ calculated from $\mathbf{k} \cdot \mathbf{p}$. 




Fig.2: The heavy hole $\mid 3 / 2,3 / 2>$ band in strained $\mathrm{Si}_{0.7} \mathrm{Ge}_{0.3}$ as grown on a $\mathrm{Si}(001)$ substrate calculated from $\mathbf{k} \cdot \mathbf{p}$.

We noted that the bandstructures shown in Figs. 1 and 2 have distinct axes of symmetry representing the underlying crystal symmetry, and the energy surfaces along these axes have an ellipsoidal like structure. These properties are used in our geometric bandstructure model since any model based on these high symmetry directions will naturally contain the correct symmetry of the valence band. The geometric model is based upon aligning non-rotational invariant ellipsoids along orthogonal vector triads based on the symmetry of the valence band. The energy surfaces for our geometric model may be written in the following form:

$$
\varepsilon_{j}(k)=\varepsilon_{0}(k)\left(\eta_{j}+\frac{1}{S_{j}}\right)
$$

where the subscript $j$ denotes the band either (1) heavy $\mid 3 / 2,3 / 2>$ or (2) light $\mid 3 / 2,1 / 2>$ and $\varepsilon_{0}(\mathrm{k})$ is the free electron energy,

$$
\varepsilon_{0}(k)=\frac{\hbar^{2} k^{2}}{2 m_{0}}
$$

$S_{j}$ has the following form:

$$
S_{j}=\frac{1}{6} \sum_{i=1}^{6}\left[\frac{\frac{1}{a_{i j}}+\frac{1}{b_{i j}}+\frac{1}{c_{i j}}}{\left(\frac{k \cdot e_{1}^{i}}{a_{i j}}+\frac{k \cdot e_{2}^{i}}{b_{i j}}+\frac{k \cdot e_{3}^{i}}{c_{i j}}\right)^{2}}\right]
$$

The parameters $\mathrm{a}_{i j}, \mathrm{~b}_{i j}, \mathrm{c}_{i j}$ may be considered to be the effective masses associated with the non-rotational ellipsoidal bands, so there is a maximum of 9 different masses. However, symmetry reduces this to 3 for the relaxed and 6 for the strained SiGe case. These may be obtained by comparing the energies calculated from our geometric model with the energy dispersion relationship as calculated from $\mathbf{k} \cdot \mathbf{p}$. The parameters $e_{1}, e_{2}, e_{3}$ are the vectors used in the triads. The following vector orthogonal triads are used here:

$$
\begin{array}{ccc}
\left(0, \frac{1}{\sqrt{2}}, \frac{1}{\sqrt{2}}\right) & \left(\frac{1}{\sqrt{2}}, \frac{1}{\sqrt{2}}, 0\right) & \left(\frac{1}{\sqrt{2}}, 0, \frac{1}{\sqrt{2}}\right) \\
\left(0, \frac{-1}{\sqrt{2}}, \frac{1}{\sqrt{2}}\right) & \left(\frac{1}{\sqrt{2}}, \frac{-1}{\sqrt{2}}, 0\right) & \left(\frac{1}{\sqrt{2}}, 0, \frac{-1}{\sqrt{2}}\right) \\
\left(\begin{array}{cc}
1,0,0
\end{array}\right) & (0,0,1) & (0,1,0) \\
\text { Triad Set } 1 & \text { Triad Set } 2 & \text { Triad Set 3 }
\end{array}
$$

Table I: Orthogonal vector triads used in the geometric model

The dispersion relationship for the spin-split off band $\mid 1 / 2,1 / 2>(3)$ is found via the completeness theorem for cubic equations [10], so that $\varepsilon_{3}(k)$ is given by :

$$
\varepsilon_{3}(k)=\varepsilon_{0}(k)\left(3 A-\eta_{1}-\eta_{2}-\frac{1}{S_{1}}-\frac{1}{S_{2}}\right)
$$

where $\mathrm{A}$ is given by:

$$
A=\frac{1}{3}(L+2 M)+\frac{\hbar^{2}}{2 m_{0}} .
$$

Here $L$ and $M$ are the usual valence band parameters found in the literature. An .example of the valence band structure calculated from our geometric model is shown in Fig.3.

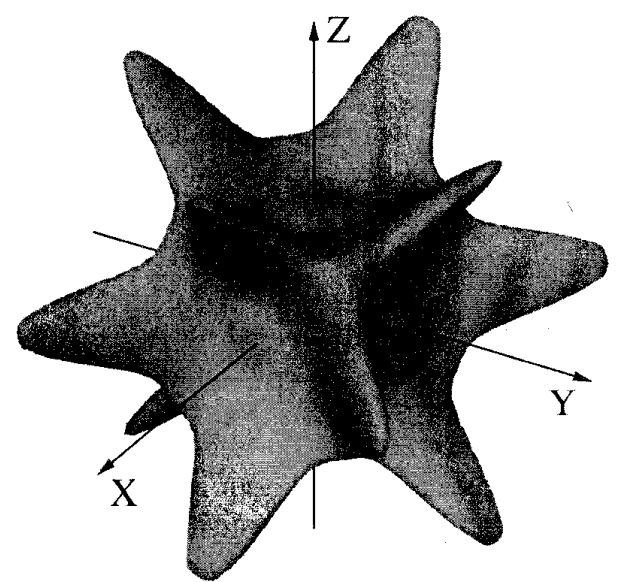

Fig.3: The heavy hole $3 / 2,3 / 2>$ band in strained $\mathrm{Si}_{0.7} \mathrm{Ge}_{0.3}$ as grown on a $\mathrm{Si}(001)$ substrate calculated from the geometric model.

There is good agreement between the bandstructures as calculated from our geometric model and the $\mathbf{k} \cdot \mathbf{p}$ results of the equivalent bandstructure shown in Fig.1. Similar agreement is obtained for the other bands. An advantage of the geometric model is that it enables us to define an 
effective mass tensor for the valence band. It also has the additional advantage that the velocities/trajectories can be calculated analytically rather than numerically which considerably speeds up Monte Carlo simulations.

\section{Transport results}

A bulk three band warped hole Monte Carlo has been developed utilising the geometric valence band model described above. The overlap integrals in the scattering matrix elements have been approximated using those given by Wiley [11] for inter and intra valley scattering involving the heavy and light hole bands, although these are only strictly valid at the zone centre. An overlap of 1 is chosen for all scattering events involving the spin-split off band. Previous simulations have neglected the inclusion of the spin-split off band [1]. However, our results show that approximately $15 \%$ of the holes occupy the spin-split off band at high fields which is comparable with the occupation in the light hole band. The calculated velocity field curves for bulk silicon and germanium are shown in Fig.4.



Fig.4: Average hole drift velocities in undoped $\mathrm{Si}$ and $\mathrm{Ge}$ at $300 \mathrm{~K}$.

We have achieved good agreement with published data $[12,13]$. A similar model can also be used for relaxed SiGe as this has the same symmetry as bulk $\mathrm{Si}$ and Ge. The mobilites calculated for relaxed SiGe from our Monte Carlo simulations are shown in Fig.5, along with the corresponding experimental data $[14,15]$. The experimental mobilities $[14,15]$ for relaxed SiGe have been used to determine the alloy scattering potential. Although the spread in the experimental data makes it difficult to find a definitive value for the alloy scattering potential we have deliberately chosen a optimistic value in order to reflect the age of the data and possible increases in mobility that may be achievable nowadays using modern fabrication techniques.

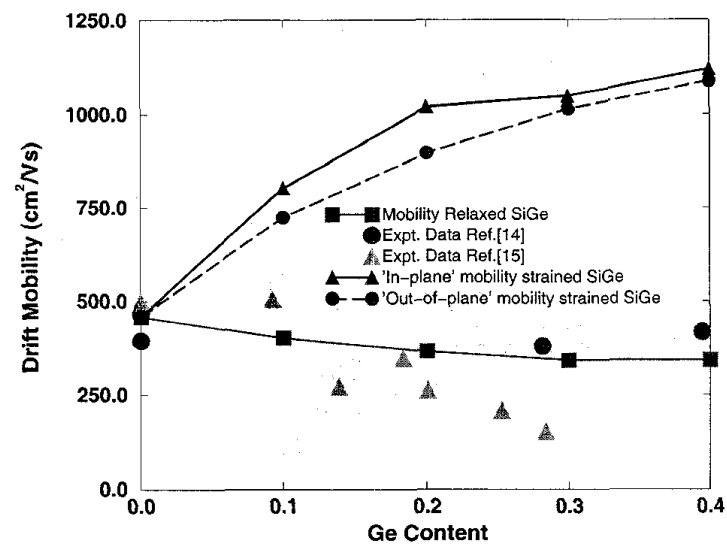

Fig. 5 Hole mobilities in relaxed and strained SiGe at $300 \mathrm{~K}$.

The geometric model has also been used to calculate the hole mobilities in 'in-plane' and 'out-of-plane' directions. 'Out-of-plane' refers to the direction 'normal to the layer', while 'in-plane' refers to directions lying on the $<001>$ plane. The mobilites for the strained $\mathrm{Si}_{1-\mathrm{x}} \mathrm{Ge}_{\mathrm{x}}$ here have been calculated assuming the same overlap integral as for the relaxed case as a first approximation in this work, although recent work has shown that the resulting change in overlap integrals due to strain may have a noticeable effect on hole transport in GaAs [16].

The results of simulations for hole mobilities in strained $\mathrm{Si}_{1-\mathrm{x}} \mathrm{Ge}_{\mathrm{x}}$ at low Germanium (0->0.4) concentrations, as this is the range which is of the most interest experimentally at the moment, are shown in Fig.5. Both the 'in-plane' and 'out-ofplane' mobilities show an improvement over unstrained Si. This is due to both a reduction in the effective masses and the strain splitting between the $\mid 3 / 2,3 / 2>$ and $\mid 3 / 2,1 / 2>$ hole bands which significantly reduces intervalley scattering between these two bands. Our studies suggest that this is the dominant mechanism for increased mobilities as in the case of electrons in strained $\mathrm{Si}$ [17]. We have not compared our simulations with experimental results since there is a noticeable dearth of experimental results for undoped strained SiGe. Experimental results for heavily doped strained layers [18-20] suffer from large experimental uncertainty making quantitative comparison impossible. The 'in-plane' mobility shows the highest mobility in agreement with other published data $[2,3]$ which is important for MOSFET operation since this represents the direction along the channel.

An area of great interest in short channel devices is the possibility of velocity overshoot within the channel. As a first approximation to investigating this we have studied ballistic, transient transport in homogeneous bulk systems. The results of our studies on this are shown in Fig. 6 for $\mathrm{Si}$ and strained $\mathrm{Si}_{0.7} \mathrm{Ge}_{0.3}$ in the 'in-plane' direction. 


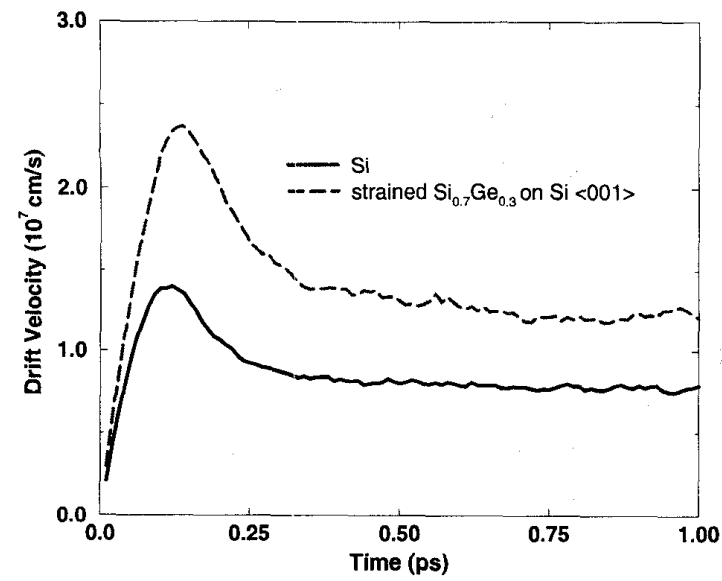

Fig.6: Transient velocity-field characteristics in $\mathrm{Si}$ and strained $\mathrm{Si}_{0.7} \mathrm{Ge}_{0.3}$ for an electric field in the $<100>$ direction.

There is clearly much greater velocity overshoot in strained $\mathrm{Si}_{0.7} \mathrm{Ge}_{0.3}$ than in relaxed $\mathrm{Si}$, although our geometric model is not intrinsically valid for high energies/fields, the general conclusions will still hold.

However, an estimate of device performance cannot be based entirely on transport data as device operation is also affected strongly by device configuration. This will effect factors such as interface scattering and ionised impurity scattering, neither of which are included in the simulations presented here. Indeed the complex energy surfaces of the valence band will give rise to complex semi-classical dynamics which may lead to adverse effects such as enhanced surface/interface roughness scattering. In particular spatially ballistic, steady state transport in heterogeneous submicron devices is fundamentally different from transient transport in bulk. It is almost impossible to make an estimate of the latter since, in the geometric valence band structure we have used, momentum $\mathbf{p}$ is not parallel to the velocity $\mathbf{v}$.

\section{Conclusions}

In this paper we have presented a simple geometric model which exhibits good agreement with the 6-band k·p analysis of the valence bandstructure in $\mathrm{Si} / \mathrm{SiGe}$ (strained and unstrained). The geometric model presented here has the advantage that within it we are able to define an effective mass tensor representation. The analytical geometric model has allowed us to develop a highly efficient Monte Carlo simulation which is suitable for use in the modelling of hole transport, both steady state and transient, in modern semiconductor devices such as $\mathrm{Si} / \mathrm{SiGe}$ MOSFETs.

\section{References}

1. T. Yamada and D.K.Ferry: Solid State Electron. 38 (1995) 885

2. M. V. Fischetti and S. E. Laux: Journal of Applied Physics 80 (1996) 2234

3. F. B. Bufler, Full Band Monte Carlo Simulation of electrons and holes in strained Si and SiGe (Herbert Utz Verlag, Wissenchaft, 1998)

4. J. M. Hinckley and J. Singh: Physical Review B 41 (1990) 2912.

5. G. Dresselhaus, A. F. Kip and C. Kittel: Physical Review 98 (1955) 368

6. J, E. Dijstra, and W. Th. Wenckebach: Journal of Applied Physics 81 (1997) 1259

7. T. Manku and A. Nathan: Journal of Applied Physics 73 (1993) 1205

8. G. Bir and G. Pikus, Symmetry and strain-induced effects in semiconductors (Wiley New York 1974)

9. M. M. Reiger and P. Vogl: Physical Review B 48 (1993) 14276

10. M. Abramowitz and I. A. Stegun, Handbook of Mathematical Functions (Dover Publications, Inc., New York 1972)

11. J. D. Wiley: Physical Review B 4 (1971) 2485

12. E. J. Ryder: Physical Review 90 (1953) 766

13. A. Alberigi Quaranta et al: Solid State Electronics 11 (1968) 685

14. G. Busch and O. Vogt: Physical Acta 33 (1960) 437

15. A. Levitas: Physical Review 90 (1955) 1810

16. J. M. Hinckley and J. Singh: J Applied Physics Letter 53 (1988) 785

17. T. Yamada, J. R. Zhou, H. Miyata and D.K. Ferry: IEEE Trans. Elec. Devices 41(9) (1994) 1513

18. Z. Matutinovic-Krstelj, V. Venkatraman, E.J. Prinz, J.C. Sturm and C.W. Magee: IEEE Trans El Dev 43 (1996) 457

19. T.K. Carns, S. K. Chun, M. O. Tanner, K. L. Wang, T. I. Kamins, J. E. Turner, D. Y. C. Lie, M.A. Nicolet and R. G. Wilson: IEEE Trans El Dev 41 (1994) 1273

20. J. M. McGregor, T. Manku , J. P. Noel, D. J. Roulston, A. Nathan and D. C. J. Houghton: Electronic Materials 22 (1993) 319 